\title{
Dossier « Politiques du changement global. Expertises, enjeux d'échelles et frontières de l'action publique environnementale " Introduction. Le global, nouvelle grandeur politique
de la nature?
}

\author{
Yann Bérard
}

Science politique, Université des Antilles, UMR 8053 Centre de recherche sur les pouvoirs locaux dans la Caraïbe (CRPLC), 97275 Schœlcher, Martinique, France

«Politiques du changement global» - la formule demande à être prise avec précaution, en indiquant au moins trois directions possibles ${ }^{1}$. Celle-ci peut, tout d'abord, désigner l'entrée en politique des changements globaux, pour reprendre ici une expression d'inspiration naturaliste, en tant que problèmes publics à caractère planétaire, dont le changement climatique est devenu, en ce début de $\mathrm{XXI}^{\mathrm{e}}$ siècle, l'une des manifestations majeures. Ce faisant, la même formule peut aussi conduire à marquer le caractère global ou transversal des changements auxquels les systèmes socioéconomiques sont confrontés face aux transformations accélérées et imprévisibles de leur environnement - ce qu'une expression comme celle de «transition écologique » aurait, pour l'heure, tendance à subsumer. Deux orientations qui, évidemment, ne sont pas sans recoupements possibles et qui pourraient même être appelées à converger en faveur de l'invention de «nouvelles » politiques publiques, à l'instar de la récente loi sur la biodiversité proposée par le gouvernement français (voir l'éditorial de ce numéro). De là, une troisième orientation peut se dégager, résultat en quelque sorte des deux premières : à savoir, l'extension globale des concepts et des méthodologies qui contribuent à problématiser les politiques environnementales, en leur donnant à la fois sens et légitimité, dont le caractère standardisé ou d'uniformité reste toutefois à déterminer. Autant d'aspects d'une question aux ramifications nombreuses et variées que l'on pourrait résumer ainsi : «quelles actions et politiques publiques face aux changements globaux ? ", dont traitent et que s'efforcent de démêler, chacune à partir d'options qui leur sont propres, les différentes contributions réunies dans ce dossier.

Ces distinctions, il est vrai, revêtent un caractère quelque peu théorique dès lors qu'on considère l'étroite relation qui se noue entre science et politique autour des enjeux qui leur sont liés. Les sciences de la nature, par exemple, mettent en avant l'impact des changements globaux sur les sociétés mais aussi des changements que ces mêmes sociétés induisent sur leur environnement, en insistant sur la nécessité d'élaborer des modèles d'analyse toujours plus complexes et sophistiqués (Gauthier-Clerc et al., 2014 ; Lagadeuc et Chenorkian, 2009 ; Le Treut, 2009; Mace, 2013). Certains travaux d'histoire et de sociologie des sciences ont bien montré, quant à eux, comment des disciplines nées de ces développements - la climatologie ou l'écologie, par exemple - avaient pu être mobilisées au travers d'alliances stratégiques et originales entre espaces professionnels, économie, droit et bureaucratie, pour donner lieu à de nouvelles valeurs de référence en matière d'action publique (Aykut et Dahan, 2015 ; Encinas de Munagorri, 2009 ; Gay, 2013 ; Vadrot,

Auteur correspondant : yberard@martinique.univ-ag.fr

Voir dans ce même numéro les autres contributions au dossier «Politiques du changement global. Expertises, enjeux d'échelles et frontières de l'action publique environnementale »: les articles de Lydie Cabane, de Marie Hrabanski, de Nicolas Rocle, de Jean-Raphaël Gros-Désormeaux, le texte de Justin Daniel et dans la rubrique « Ouvrages en débat », la contribution de Lydie Cabane.

1 La réalisation de ce dossier a bénéficié du soutien des «Investissements d'Avenir » de l'Agence nationale de la recherche française (Ceba, réf. ANR-10-LABX-25-01). 
2014). Par les questions qu'elles posent, les indicateurs qu'elles forgent et les équipements qu'elles utilisent, les sciences de la nature peuvent ainsi, au même titre que les sciences sociales, apporter une contribution décisive à la définition des régimes d'action et de vérité du monde social.

C'est avec le désir d'approfondir ces pistes d'analyse et de réflexion que le thème de ce dossier a été conçu ${ }^{2}$. Soucieux de considérer l'horizon aujourd'hui explicitement politique des sciences et des techniques (qu'elles soient sociales, de la nature ou du génie), le dossier s'intéresse à la façon dont les changements environnementaux dits "globaux ${ }^{3}$ » affectent tant l'action des pouvoirs publics que celle des individus à l'ère de " l'Anthropocène » (Bonneuil et Fressoz, 2013 ; Chartier et Deléage, 2010 ; Garrard et al., 2014 ; Gemenne, 2015 ; Hache, 2014 ; Sinaï, 2013 et 2015). Sans nécessairement chercher à trancher les controverses autour de la nature (anthropique ou capitalistique, inéluctable ou remédiable, etc.) de ces changements, les articles rassemblés pour l'occasion s'efforcent avant tout d'explorer la manière même dont les transformations qu'ils désignent sont conçues, formulées, critiquées, amendées, rectifiées, validées, appropriées ou rejetées, dans des contextes sociopolitiques situés. Il s'agit bien par là, non pas « de dénier l'importance de la production conceptuelle dans la dynamique des sciences de l'environnement, et dans celle des pratiques collectives, mais d'en appeler à plus de réflexivité sur leur contenu et leur association » (Petit et al., 2014, p. 188).

On peut relever, à cet égard, que les préoccupations associées à ces changements s'expriment désormais clairement aux échelons politique et institutionnel international et européen (Conventions-cadres des Nations unies sur les changements climatiques, pour la diversité biologique et sur la lutte contre la désertification, Convention de Stockholm sur les polluants organiques persistants, Paquet énergie-climat ou Stratégie de l'Union européenne pour la diversité biologique, etc.) comme aux niveaux national ou local (Grenelle de l'environnement, Stratégie nationale de la biodiversité ou de la transition écologique, plans d'actions régionaux et urbains, etc.). Ces préoccupations interpellent, en outre, directement l'opinion publique et l'action des individus, non seulement dans leurs modes de vie quotidiens mais aussi, et

\footnotetext{
2 Celui-ci a notamment profité des échanges menés dans le cadre du séminaire "Changements environnementaux et société » animé par l'équipe émergente « Biodiversité, environnements, sociétés, territoires » du Centre de recherche sur les pouvoirs locaux dans la Caraibe (UMR 8053), dont nous tenons à remercier ici l'ensemble des participants.

3 Qu'ils s'énoncent sous la forme de dérèglement climatique, d'érosion de la diversité biologique, de dégradation des sols, de pollution chimique de l'air ou de l'eau, d'usage intensif des ressources hydriques, etc.
}

peut-être surtout, lorsque ceux-ci sont confrontés à des " catastrophes » (Aradau et van Munster, 2011) ou à des «événements extrêmes » (Décamps et Mathieu, 2005 ${ }^{4}$. En trame de fond, se fait ressentir l'exigence de plus en plus pressante d'un modèle économique et social qui renouvelle nos façons de consommer, de produire, de travailler et de "vivre en commun », ainsi que l'illustrent, par exemple, les travaux menés depuis une vingtaine d'années sur la modernisation écologique (Mol et al., 2009) ou, plus récemment, sur l'économie circulaire (Dubuisson-Quellier, 2014).

La connaissance autant que l'incertitude, associées aux effets potentiels ou avérés des changements globaux, génèrent ainsi des attentes et des politiques environnementales qui affichent une grande ambition, avec la volonté de changer nos pratiques actuelles et, plus largement, les valeurs de référence qui structurent les relations entre systèmes naturels et systèmes sociaux (Flipo, 2014 ; Hache, 2011 ; Harribey, 2013 ; Larrère et Larrère, 2015 ; Mairet, 2012 ; Maris, 2010). Sous ces différents aspects, l'analyse des «politiques du changement global » se montre inséparable d'une réflexion sur les fondements scientifiques et techniques de la civilisation moderne, aussi bien que sur les effets d'une conception à dominante marchande ou gestionnaire de la nature, telle qu'on peut notamment la retrouver formulée en termes de «développement durable» (Rumpala, 2010) ou de «croissance verte » (Gaudillière et Flipo, 2009). De là découle tout un ensemble de techniques de gouvernement que les différentes contributions réunies dans ce dossier se proposent d'examiner de plus près.

\section{Un « tournant global » des politiques environnementales?}

Au regard de ces éléments de contexte, l'importance prise par la thématique des changements globaux dans de nombreux domaines d'action publique ainsi qu'à tous les échelons de gouvernement invite à se demander si les politiques d'intervention environnementale $n^{\prime}$ ont pas elles-mêmes connu un «tournant global » irréversible. Telle est l'une des interrogations de fond qui motive la constitution de ce dossier et peut rejoindre, sous un angle plus réflexif (on pourrait même dire «méta-réflexif »),

4 Le cas de l'opinion française est illustratif, dans le contexte de préparation de la prochaine Conférence des parties sur le climat, puisqu'à la question "Quand vous entendez parler de changement climatique, quels termes correspondent le mieux à ce que vous pensez ?», les Français répondent d'abord « urgence » $(52 \%)$, puis «mobilisation » $(30 \%)$. Cf. COP21 : les Français face au changement climatique, enquête Ipsos/Havas, février 2015, http://fr.slideshare.net/IpsosFrance/cop21-lesfranais-face-au-changement-climatique-ipsos-havas-paris. 
la mise en procès d'une ontologie naturaliste prédominante (Descola, 2011). Car le questionnement repose, en réalité, sur un double présupposé, dont la corrélation mérite d'être interrogée : celui de l'échelle, d'une part, qui consisterait à dire qu'on ne saurait plus désormais gouverner l'environnement indépendamment d'une problématique globale ; celui de la culture, d'autre part, qui permettrait de connaître cet environnement - d'un point de vue naturaliste, il s'agit là, bien sûr, d'une culture à la fois matérielle et objectivante. Dans cette perspective, on est aussitôt amené à se demander s'il est encore possible de penser «la Nature» en dehors de l'interprétation et de l'utilisation des résultats de la recherche scientifique et technique, mais aussi d'objectifs politiques, économiques et juridiques. À cela pourraient encore s'ajouter les implications spatiales et territoriales de ces choix, les modes d'administration et de gestion à privilégier, voire la façon dont les sciences humaines et sociales elles-mêmes sont équipées afin d'étudier ces processus et d'en rendre compte (Latour, 2012 ${ }^{5}$ ).

Ces questionnements engagent un effort de définition plus poussé quant au sens et à la substance qu'il convient de prêter aux politiques environnementales. Trouvant leur origine dans la montée en puissance et les " excès » de la révolution industrielle au cours de la seconde moitié du XIX ${ }^{\mathrm{e}}$ siècle, les politiques d'intervention environnementale ne prennent leur essor véritable, il y a environ cinquante ans, que sous l'effet des pratiques et des critiques de l'économie productiviste d'après-guerre, encourageant une rationalisation toujours plus poussée du vivant et des activités humaines sur Terre (Fressoz et al., 2014). Dans ces conditions, les politiques publiques ayant pour objet « l'environnement » peuvent se caractériser par une prolifération de domaines d'intervention (du patrimoine architectural aux énergies renouvelables en passant par les OGM et la biodiversité) et des formes de régulation multiples (juridique, fiscale, économique, conventionnelle, interactive ou « participative »). Elles se distinguent également par des enjeux qui, le plus souvent, se montrent rétifs aux découpages territoriaux administratifs traditionnels, faisant de l'articulation des échelles spatiales un défi concret à relever pour l'action publique (Lascoumes, 2012). De ce fait, l'environnement est devenu, au XXe siècle, un objet «complexe », dont l'autonomie autant que la gouvernabilité demeurent sujettes à caution : transversal à de nombreux secteurs

\footnotetext{
5 On relèvera que la plupart de ces considérations se trouvent au centre des interrogations ou controverses ayant conduit à l'institutionnalisation ou à la réforme des activités et procédures d'organismes tels que le Giec (Groupe d'experts intergouvernemental sur l'évolution du climat) ou, plus récemment, l'IPBES (Intergovernmental science-policy platform on biodiversity and ecosystem services; en français, Plateforme intergouvernementale sur la biodiversité et les services écosystémiques ) (Beck et al., 2014).
}

d'activités (énergie, transport, agriculture, santé, tourisme, etc.) et niveaux de gouvernement (urbain, national, européen, international, etc.), il combine des questions tant scientifiques qu'économiques, juridiques et politiques, où la connaissance fait rarement l'unanimité, soit en raison d'intenses controverses, soit parce que se révélant, tout simplement, incomplète ${ }^{6}$. De cette complexité accrue résultent des décisions difficiles à prendre et des programmes d'action publique dont les objectifs demeurent flous et ambivalents.

Ces caractéristiques apparaissent encore renforcées par l'émergence d'une nouvelle catégorie de menaces dites «globales », qu'illustrent assez bien l'impact du $\mathrm{CO}_{2}$ et des autres gaz à effet de serre sur le réchauffement climatique ou celui des activités humaines sur la dégradation de la biodiversité (Aubertin, 2014) - avec leurs effets en chaîne ou croisés (migratoires, sanitaires, etc.) (Cournil et Vlassopoulos, 2015; Guégan et Renaud, 2005), potentiels ou avérés. La géopolitique, si l'on peut dire, de ces menaces renvoie également à une généalogie particulière, qui montre ici tout l'intérêt de penser de concert, sans les séparer mais en les articulant, changements environnementaux et changements culturels, techniques ou sociaux (Dahan, 2007 ; Grevsmühl, 2014 ; Mahrane et al., 2012). Or les situations de risque et d'incertitude qui en découlent présentent des particularités que les critères habituels de prise de décision politique ont encore du mal à appréhender, avec pour effet, sinon de reporter les actions à entreprendre, du moins de les freiner, comme l'illustre le cas du climat (Giddens, 2011 ; Urry, 2011 ; Zaccai et al., 2012) : phénomènes peu visibles par excellence, résultat d'interactions multiples, pour lesquelles une responsabilité individuelle est difficile à imputer, ces menaces sont souvent perçues de manière abstraite, brouillant l'urgence en la matière ; elles sont inséparables, en outre, de questions où les controverses scientifiques prolifèrent, parfois orientées, voire manipulées par des intérêts économiques ou financiers, et relayées par les médias ; elles nécessitent, malgré tout, des prises de décisions en situation d'incertitude, dont les effets ne se feront ressentir qu'à moyen ou à long terme, rendant par définition toute évaluation difficile, voire impossible, à court terme - une temporalité qui apparaît en décalage profond avec celle de la « politique électorale » (par opposition à la «politique des problèmes » [Leca, 1996]), et plus généralement avec celle des questions économiques qui dominent les agendas gouvernementaux tout autant que ceux de la vie quotidienne au sein des démocraties de marché occidentales, conduisant à relativiser certains effets de la conjoncture

\footnotetext{
6 Témoigne de ces intrications, au plan académique, par exemple, la diversité des options sociologiques développées au cours de ces trente dernières années dans l'espace francophone (Barbier et al., 2012).
} 
médiatico-politique ${ }^{7}$; ces menaces, enfin, appellent une nécessaire (ce qui ne veut pas dire suffisante) prise en charge à l'échelon international, là où la mobilisation a toujours été marquée par la lenteur ou l'irrégularité des avancées et par un engagement très variable, pour ne pas dire foncièrement inégal, des États. Après avoir rapidement gagné en extension au cours des deux dernières décennies, la problématique du climat apparaît ainsi confrontée à la nécessité de trouver une nouvelle consistance politique, qui réclame la constitution de nouveaux axes d'argumentation (Szerszynski et Urry, 2010). Loin de dépeindre un retour en arrière, cette orientation vers une « repolitisation » du problème suppose, au contraire, la concrétisation d'objectifs restés jusque-là en grande partie abstraits, ou ce que l'on pourrait également convenir de nommer une « montée en singularité ${ }^{8}$ ». Décrivant l'une des dimensions du travail de création artistique, l'expression pourrait aussi renvoyer à un certain « art de gouverner », qui appelle au renouvellement des formats d'action publique et collective ${ }^{9}$.

Dans la conjoncture actuelle, l'émergence de ces menaces globales met plus particulièrement en évidence les tensions multiples auxquelles doivent faire face les politiques d'intervention environnementale: qu'il s'agisse de la nouveauté des problèmes à traiter (inventorier et réguler les "socioécosystèmes »), de la part d'incertitude concernant les risques encourus (leur ampleur et leur vitesse notamment), des dynamiques spatiales globales ou transfrontalières impliquées au même titre que la divergence des intérêts en présence (entre pays du Nord et pays du Sud, par exemple) (Boisvert et Vivien, 2010), ou du degré de priorité à accorder aux questions environnementales par rapport à d'autres enjeux de société (emploi, éducation, immigration, etc.). À cela, conviendrait-il d'ajouter l'ensemble des évolutions, des critiques ou des formes d'alternatives, plus ou moins radicales, regardant nos manières de

7 Notons que selon une enquête menée hors du contexte de la COP21, l'environnement, avec $9 \%$, arrive en avant-dernière position des préoccupations des Français, loin derrière les trois premières : le chômage (56\%), les impôts et les taxes (43\%), et le pouvoir d'achat (36\%). Cf. France 2014 : les nouvelles fractures, enquête Ipsos/Steria pour Le Monde, France Inter, Fondation Jean-Jaurès et le Cevipof, janvier 2014, http://www. franceinter.fr/sites/default/files/2014/01/20/821768/fichiers/ Barom\%C3\%A8tre\%20nouvelles\%20fractures_2014\%20vDEF.pdf.

8 Pour reprendre ici une expression de Nathalie Heinich (2004, p. 73), à propos de la sociologie de l'art.

9 À l'instar de certaines des idées lancées par Bruno Latour, comme celle d'impliquer et de chercher à représenter les "non-humains » dans les négociations internationales sur le climat. Cf. Make it work/Le théâtre des négociations, un projet de Sciences Po et du théâtre Nanterre-Amandiers, 29-31 mai 2015, http:/ / www.nanterre-amandiers.com/2014-2015/make-it-workle-theatre-des-negociations/. concevoir la solidarité (Dardot et Laval, 2014 ; Gadrey, 2015 ; Humbert et al., 2011 ; Löwy, 2008), d'habiter, de travailler ou de se nourrir sur la planète (Deverre et Lamine, 2010 ; Gadéa, 2015 ; Theys, 2011). Tout cela constitue autant de variables qui contribuent à configurer les «politiques du changement global» dans la période actuelle - soit l'action autant que l'inaction en la matière.

Sur ces différents points, il existe désormais une littérature abondante, quoique surtout anglophone, consacrée aux défis d'une gouvernance globale de l'environnement ${ }^{10}$. La construction d'un référentiel global des politiques environnementales, en tant que vision du monde à partir de laquelle les acteurs organisent leur perception du problème, confrontent leurs solutions et définissent leurs propositions d'action (Muller, 2013), n'a cependant pas fait jusqu'à présent l'objet d'une attention systématique. D'importants chantiers de recherche convergent aujourd'hui en direction de ce questionnement, notamment à travers les impératifs liés à une refonte des institutions à l'échelle internationale - synthétisés sous le label «Earth System Governance » (Biermann et al., 2012) - et à travers les défis associés à une cosmopolitique des risques (Beck, 2009), en particulier à partir du régime climatique (Jasanoff, 2011). D'autres pistes d'investigation, certes moins ambitieuses par les objectifs qu'elles affichent ou les moyens qu'elles mettent en œuvre, fournissent cependant des clés de lecture, elles aussi essentielles à la compréhension des processus de globalisation des politiques environnementales. C'est en particulier le cas des études menées depuis quelques années, en France, sur les formes alternatives d'expérimentation et de conceptualisation du risque et des vulnérabilités (Boudia et Henry, 2015 ; Bourg et al., 2013 ; Revet et Langumier, 2013 - voir la recension de cet ouvrage dans ce numéro) ou encore sur les dynamiques de circulation des normes environnementales (Alphandéry et al., 2012). Notons, enfin, l'originalité de certaines recherches conduites dans le champ des postcolonial studies sur les «écologies globales » (DeLoughrey et al., 2015), ainsi que les efforts non négligeables déployés au cours de ces dernières années par certains spécialistes des sciences de l'environnement pour faire advenir des récits inédits sur les liens entre écosystèmes et "systèmes-monde " (Hornborg et al., 2007).

\footnotetext{
10 Quel'on songe, par exemple, aux nombreux travaux interdisciplinaires de sciences sociales publiés par la revue Global Environmental Change, créée en 1990 dans un contexte marqué par la mise à l'agenda politique des préoccupations environnementales à l'échelle planétaire (une de ses rubriques, UNU Monitor, fut consacrée pendant dix ans aux recherches de l'Université des Nations unies). Citons aussi Global Environmental Politics, lancée en 2001 par les presses du MIT, à l'initiative de Peter Dauvergne, qui prend explicitement acte des dimensions politiques du changement global (en particulier climatique).
} 


\section{Le changement global à l'épreuve des sciences et du politique}

Prenant en considération ces avancées et ces limites, le dossier propose une analyse approfondie du travail de production de l'expertise et de légitimation de l'autorité politique qui oriente et met à l'épreuve une définition "globale » de l'action publique environnementale dans différentes régions $\mathrm{du}$ monde. Plus que dans d'autres domaines d'intervention publique, les liens entre connaissance et action, expertise et décision, ont toujours constitué un paramètre-clé en matière d'environnement (Forsyth, 2003), cela singulièrement des deux côtés de l'Atlantique (Jasanoff, 2005). Un tel projet justifie une démarche à la fois comparative et réflexive, mobilisant en l'occurrence les apports conjoints de la science politique, de la sociologie, de la géographie ou de l'anthropologie des sciences et des techniques.

Dans cette optique, l'expertise peut constituer un bon outil d'analyse des « politiques du changement global ». C'est là, tout d'abord, l'un des intérêts de la contribution de Lydie Cabane, qui ouvre ce dossier, que de souligner la pluralité des options épistémiques à l'œuvre entre Europe, Afrique et Amérique dans la définition des " catastrophes ", dont elle questionne la dimension paradigmatique pour lire et comprendre 1'actuelle « globalisation environnementale ». Avec la montée en puissance des préoccupations pour la biodiversité, le rapprochement des savoirs de l'écologie avec ceux de l'économie - incarné par le succès de certaines thèses issues du courant de l'économie écologique (ecological economics) dans les années 1990 - a pu également constituer un ressort puissant d'expérimentation politique, comme en témoignent la genèse centraméricaine puis l'internationalisation de l'instrument « paiements pour services environnementaux » dont se fait l'écho, à sa suite, l'article de Marie Hrabanski. Mais l'expertise peut être encore appréhendée dans sa capacité même à cadrer ou à problématiser l'action publique face aux changements globaux, comme le montrent les trois autres contributions au dossier. L'enquête de Nicolas Rocle, consacrée aux défis d'une adaptation au « risque climat » sur les littoraux aquitain et martiniquais, souligne toute l'importance de certaines organisations-frontières pour inscrire le mot d'ordre d'adaptation sur les agendas politiques locaux. De même, dans un contexte pas si éloigné, JeanRaphaël Gros-Désormeaux rend compte des incertitudes dont tentent de s'extraire les experts pour évaluer la biodiversité dans les Antilles françaises, à travers l'analyse d'un dispositif aujourd'hui devenu clé pour étudier les continuités écologiques de ces territoires, les Zones naturelles d'intérêt écologique, faunistique et floristique (Znieff). Une fonction de cadrage que l'on retrouve encore dans la contribution, centrée à la fois sur les discours et les politiques, de Justin Daniel (rubrique «Vie de la recherche »), lorsque ce dernier insiste sur la façon dont l'impératif de développement durable met à l'épreuve l'organisation politique d'une région comme celle de la Caraibe, réputée tant pour la diversité que pour la fragilité de ses écosystèmes. À ce titre, l'approche retenue dans ce dossier enjoint à mettre en regard des situations contrastées, sans se focaliser uniquement sur les démocraties des pays d'industrialisation ancienne, à travers la prise en compte de différentes aires régionales et, le cas échéant, de leurs interrelations (ici entre l'Afrique, les Amériques, la Caraibe ou l'Europe), tout en faisant varier les échelles d'observation. De cette manière, le dossier appelle à contribuer aux enjeux scientifiques qui s'attachent à l'étude et aux définitions $\mathrm{du}$ « global ».

Chercher à saisir la globalité d'un phénomène peut s'envisager à partir d'un espace géographique ou social, selon une perspective d'analyse relationnelle ou substantielle (Pries, 2005). L'entreprise suppose, en outre, un espace interdisciplinaire de réflexion et de partage, qui jusqu'à présent a peu pénétré l'espace académique français des sciences sociales, par contraste avec l'intérêt croissant de la littérature anglophone pour les global studies $^{11}$. C'est aussi à renouer avec cet esprit de dialogue et de réflexivité que s'essaient les textes ainsi réunis. Sur ce point, là encore, les contributions affichent une diversité de choix possibles : que ce soit, comme le fait L. Cabane, en signalant le caractère relatif et parfois exclusif de la circulation des idées notamment produites par les sciences sociales en matière de " catastrophes »; ou bien tel que le donne à voir M. Hrabanski, lorsqu'elle insiste sur le rôleclé joué par certains experts dans la diffusion internationale de l'instrument « PSE », dont le caractère $\mathrm{d}^{\prime}$ « instrument de marché » fait débat. Une autre option consiste alors à mettre davantage l'accent, comme le font $\mathrm{N}$. Rocle ou J.-R. Gros-Désormeaux, sur le travail de traduction que sont appelés à réaliser certains acteurs pour parvenir à en convaincre et à en mobiliser d'autres ; ou encore, dans la perspective suivie par J. Daniel, à mettre en place des dispositifs d'action publique qui articulent à la fois cohésion et ouverture régionale. Dans cette optique, le dossier invite à une reconnexion des travaux portant sur

\footnotetext{
11 Malgré l'existence de travaux relevant des problématiques du global en anthropologie, en géographie, en histoire et en sociologie, Alain Caillé et Stéphane Dufoix (2013) voient dans la difficulté à faire émerger en France un « espace transdisciplinaire " sur cette question, deux raisons : d'une part, l'assimilation du « global » à la mondialisation comme phénomène purement économique et financier, voire comme manifestation de l'impérialisme culturel américain, a cantonné les recherches à une rhétorique de la dénonciation ; de l'autre, une organisation de la recherche et de l'enseignement supérieur fondamentalement "disciplinaire » a empêché le rassemblement de chercheurs et d'enseignants et favorisé le repli des associations professionnelles.
} 
la gouvernance globale de l'environnement et les cosmopolitiques du risque avec une analyse sociopolitique de la circulation des savoirs et de leur appropriation dans des contextes situés (Jasanoff et Long Martello, 2004).

Plus qu'une discussion académique autour de la gouvernance de l'environnement, les arbitrages que les sociétés modernes se doivent aujourd'hui de rendre à échéance régulière sur les changements environnementaux qui les affectent (notamment en matière d'énergie, de climat ou de biodiversité) ne peuvent que s'enrichir des analyses menées en termes d'action et de politiques publiques. Tel est, également, l'un des choix privilégiés par les contributeurs de ce dossier, sur lequel il convient d'insister : qu'il s'agisse des présupposés à l'œuvre dans les «politiques du risque », dont $\mathrm{L}$. Cabane déconstruit le «mythe européen unificateur» avant de le mettre en regard avec $\mathrm{d}^{\prime}$ autres approches dans une optique d'histoire croisée; ou des politiques nationales costaricaines ayant donné naissance aux premiers «PSE », relayées par certaines grandes organisations internationales ou ONG de conservation, que l'on retrouve au centre de l'enquête de M. Hrabanski. Il s'agit aussi, à une échelle locale, des politiques climatiques littorales qu'analyse, comparaison à l'appui, N. Rocle, ou des politiques de patrimonialisation de la biodiversité dont se saisit J.-R. Gros-Désormeaux sous l'angle des pratiques expertes; sans oublier les enjeux d'un "régionalisme ouvert » pour le bassin caribéen qu'expose J. Daniel, en termes de développement durable. Cette orientation pragmatique de la recherche, si elle n'est pas toujours allée de soi en France, notamment au regard d'une tradition des sciences sociales anglophones sans doute moins frileuses (Kalaora et Vlassopoulos, 2013), constitue désormais une préoccupation légitime pour un nombre croissant d'acteurs ou de publics concernés (chercheurs, militants, citoyens, élus, consultants, journalistes, gestionnaires, etc.), qui, chacun à leur manière, si l'on peut dire, "mènent l'enquête ». Outre les enjeux socioéconomiques importants qu'elles comportent, les «politiques du changement global » interpellent alors aussi directement le droit, lorsque se trouvent impliqués des publics marginalisés ou peu en prise avec les dispositifs de pouvoir (" générations futures », « peuples autochtones », « espèces menacées », etc.), dont juristes comme sociologues soulignent combien la représentation demeure incertaine et fragile (Deldrève et Candau, 2015 ; Hermitte, 2011 ${ }^{12}$ ).

\footnotetext{
12 En ce cas, on dira que la prise en compte des «nonhumains » dans l'analyse (qu'il s'agisse d'animaux, de plantes, de molécules, d'artefacts, etc.) nécessite de faire jouer un principe de symétrie " modeste » entre acteurs humains et entités non humaines, qui s'applique avant tout aux plans méthodologique et épistémologique, mais qu'on ne suivra pas forcément au plan ontologique (Granjou, 2013).
}

Ce faisant, une telle perspective de recherche se montre inséparable d'une attention toujours plus poussée en direction des logiques micropolitiques, à la fois expérimentales et transversales, qui irriguent la fabrique du «global » en matière d'environnement (Foyer, 2010 ; Granjou, 2013 ; Labatut, 2010 ; MartinezAlier, 2002 ; Poirot-Delpech et Raineau, 2012 ; Selmi et Hirtzel, 2007). Dans ces conditions, on ne saurait parler qu'au singulier des enjeux de l'énergie, du climat ou de la biodiversité, par exemple ${ }^{13}$. C'est le parti pris auquel les textes ici rassemblés invitent, une fois de plus, en rappelant, tout d'abord, comme le fait L. Cabane, l'ancrage sociopolitique des «ontologies du global » à l'œuvre dans les disaster studies, dont les théories ne sauraient être restituées indépendamment du contexte qui les a vues naître. C'est aussi la démarche suivie par M. Hrabanski lorsqu'elle revient sur la trajectoire imprévisible des « PSE » à l'international, dont le succès tient surtout à la rencontre d'une fenêtre d'opportunité politique exceptionnelle. Cette attention prêtée à la singularité des situations apparaît encore nécessaire pour décrypter, comme $\mathrm{s}^{\prime} \mathrm{y}$ attache N. Rocle, la complexité des politiques locales d'adaptation au changement climatique; de même que les processus d'évaluation et de « mise en visibilité » de la biodiversité étudiés par J.-R. Gros-Désormeaux amènent à considérer, à côté de situations fortement codifiées bien que souvent à distance des territoires, les situations peu formalisées mais ancrées territorialement, où l'expérimentation d'un savoir en quête de reconnaissance tant sociale que scientifique est rendue possible. Un genre de situation que l'on peut également mettre en parallèle, à une autre échelle, avec l'empilement des «modes de gouvernance » qui caractérise l'espace régional caribéen, dont J. Daniel rappelle que l'existence politique demeure à inventer, tout particulièrement en matière d'intervention environnementale. De cette manière d'appréhender la "globalisation environnementale», pour reprendre ici une expression de L. Cabane, résulte une image du "global» qui ne s'oppose pas au «local», pas plus qu'elle n'opposerait entre eux les échelons d'intervention, mais qui inscrit à chaque fois les changements auxquels les sociétés sont confrontées dans une dynamique transversale, traçant comme une oblique, irréductible à une seule manière de connaître comme de gouverner - tel est certainement, au passage, l'un des enseignements majeurs que l'on peut retenir du dossier.

Tenant compte de ce pluralisme inhérent à la fabrique «politique » des changements globaux, l'objectif de ce dossier appelle plus largement à rendre compte des

\footnotetext{
13 Sur ce point, les conférences de négociations internationales, très médiatisées, et même "décentralisées ", ne constituent qu'un instrument parmi d'autres, dont l'efficacité pour traiter d'enjeux globaux aussi complexes reste à démontrer (Andresen, 2012).
} 
formes de problématisation qui placent l'environnement dans le «jeu du vrai et du faux» de notre époque (Foucault, 2001) et l'érigent à la fois en percept, concept et précepte (Micoud, 2005). Les changements environnementaux globaux ont-ils suscité des formes d'action publique inédites ou ne font-ils que remettre au goût du jour de «vieilles questions»? Telle pourrait être, au fond, une autre manière de formuler le problème.

\section{Expertises, enjeux d'échelles et frontières de l'action publique environnementale}

Les présentes contributions, qui ne couvrent qu'une partie des changements environnementaux et de leurs modes d'appréhension ou de régulation, engagent, pour apporter des éléments de réponse à cette question, une réflexion que l'on peut schématiquement résumer à trois niveaux: (i) la conduite des politiques environnementales (posant ou, plus exactement, reposant la question «qui gouverne? »-ou, à tout le moins, « qui oriente les prises de décisions publiques ») ; (ii) le positionnement de l'intervention (« quelle est [ou quelles sont] la [ou les] «bonne[s] » échelle[s] d'action publique et de gouvernement ? ») ; (iii) le contenu de ce qui est proposé (« qu'estce qui fait ou ferait aujourd'hui l'unité ou la qualité des politiques environnementales?»).

En considérant chacune de ces interrogations et les incertitudes qui les traversent, plusieurs entrées analytiques peuvent être à leur tour distinguées, dont chaque article offre un traitement particulier: l'expertise, tout $\mathrm{d}^{\prime}$ abord, qu'elle soit d'inspiration savante, militante ou profane, associant sciences sociales, de la nature ou du génie, dont le rôle se veut essentiel dans l'évaluation, la sélection et la hiérarchisation des enjeux environnementaux ; les jeux d'échelles ou dynamiques transscalaires ${ }^{14}$, ensuite, dont la prise en compte et l'importance déterminent fortement sur ce sujet, en retour, à la fois les manières de connaître et celles de gouverner, la constitution des problèmes et celle de leurs modes de résolution ; les frontières de l'action publique environnementale, enfin, qui apparaissent sur ces deux plans particulièrement mises à l'épreuve face à l'émergence de menaces globales, incluant non seulement la circulation des concepts et des méthodologies, d'un territoire ou $\mathrm{d}^{\prime}$ un secteur à un autre, mais aussi leurs modalités d'appropriation, d'adaptation ou de contournement, de rejet ou d'extraversion, suivant une optique plus culturaliste (Macnaghten et Urry, 1998 ; Yearley, 2005). Telles sont les trois grandes entrées, dont l'articulation offre ici un outil d'analyse souple mais ferme, retenues dans le cadre de ce dossier pour se saisir et rendre compte des

\footnotetext{
14 Qui impliquent avant tout de prêter attention à la «structure feuilletée du social », selon la formule de Jacques Revel (1996, p. 13).
}

transformations et des invariants de l'action publique environnementale face à la problématique des changements globaux.

Poser la question d'un «tournant global » des politiques environnementales suppose dès lors de mettre à jour une pluralité de dispositifs, $\mathrm{d}^{\prime}$ « arts de faire » comme de modalités d'énonciation, dont il serait bien présomptueux de prétendre percer l'agencement et identifier les forces en présence sur la base d'un critère simple. C'est pourquoi, à défaut de pouvoir toujours livrer des explications systématiques sur la réalité des phénomènes étudiés, l'effort de réflexivité engagé plaide en faveur d'une mise en résonance de situations variées, susceptible d'en offrir une meilleure intelligibilité d'ensemble - à l'instar d'une démarche ethnographique multisituée (Marcus, 1995). Pour examiner ces situations et mieux en cerner les contours, les articles de ce dossier présentent des enquêtes qui sont le fruit d'une réflexion collectivement approfondie. Dans le même temps, plusieurs d'entre eux accordent un intérêt spécifique aux enjeux du changement climatique et de la biodiversité, là précisément où les processus de mise en politique(s), c'est-à-dire les modes d'accès à l'espace politique et de construction de ces enjeux en problèmes d'action publique et de gouvernement, sont à la fois les plus anciens et les plus actuels ${ }^{15}$. Sur la base d'études de cas et de comparaisons empruntant à des contextes géographiques et sociopolitiques diversifiés, les contributions rassemblées pour l'occasion délivrent tour à tour des récits aux perspectives de recherche originales, qui appellent chacune des discussions et des prolongements, sans prétendre en épuiser la matière.

\section{Références}

Alphandéry, P., Djama, M., Fortier, A., Fouilleux, È. (Eds), 2012. Normaliser au nom du développement durable, Versailles, Quæ.

Andresen, S., 2012. Do we need more global sustainability conferences?, in Dauvergne, P. (Ed.), Handbook of global environmental politics, Cheltenham, Edward Elgar, 87-96.

Aradau, C., van Munster, R., 2011. Politics of catastrophe. Genealogies of the unknown, New York/London, Routledge.

\footnotetext{
15 Ce dossier s'inscrit dans le prolongement de dossiers de NSS auxquels il vient apporter un regard et des perspectives complémentaires. Parmi les dossiers déjà publiés, citons notamment " Adaptation aux changements climatiques » (18, 3, 2010), "Écologisation des politiques publiques et des pratiques agricoles » $(21,2,2013)$, «Territoires en transition environnementale » $(22,3,2014)$, ou, comme l'actualité y invite, «Les enjeux de la conférence de Paris. Penser autrement la question climatique»(23, supplément 2015), ainsi que les contributions régulièrement consacrées au suivi des négociations internationales (Poznań, Copenhague, Nagoya, etc.) que l'on peut retrouver dans d'autres numéros.
} 
Aubertin, C., 2014. Biodiversité et développement durable : un couple fusionnel, in Gauthier-Clerc, M., Mesléard, F., Blondel, J. (Eds), Sciences de la conservation, Louvain-laNeuve, De Boeck, 271-277.

Aykut, S.C., Dahan, A., 2015. Gouverner le climat? 20 ans de négociations internationales, Paris, Presses de Sciences Po.

Barbier, R., Boudes, P., Bozonnet, J.-P., Candau, J., Dobré, M., Lewis, N., Rudolf, F. (Eds), 2012. Manuel de sociologie de l'environnement, Québec, Presses de l'Université Laval.

Beck, U., 2009. World at risk, Cambridge, Polity Press.

Beck, S., Borie, M., Chilvers, J., Esguerra, A., Heubach, K., Hulme, M., Lidskog, R., Lövbrand, E., Marquard, E., Miller, C., Nadim, T., Neßhöver, C., Settele, J., Turnhout, E., Vasileiadou, E., Görg, C., 2014. Towards a reflexive turn in the governance of global environmental expertise. The cases of the IPCC and the IPBES, GAIA, 23, 2, 80-87.

Biermann, F., Abbott, K., Andresen, S., Bäckstrand, K., Bernstein, S., Betsill, M.M., Bulkeley, H., Cashore, B., Clapp, J., Folke, C., Gupta, A., Gupta, J., Haas, P.M., Jordan, A., Kanie, N., Kluvánková-Oravská, K., Lebel, L., Liverman, D., Meadowcroft, J., Mitchell, R.B., Newell, P., Oberthür, S., Olsson, L., Pattberg, P., Sánchez-Rodríguez, R., Schroeder, H., Underdal, A., Camargo Vieira, S., Vogel, C., Young, O.R., Brock, A., Zondervan, R., 2012. Navigating the anthropocene: improving earth system governance, Science, 335, 6074, 1306-1307.

Boisvert, V., Vivien, F.-D., 2010. Gestion et appropriation de la nature entre le Nord et le Sud. Trente ans de politiques internationales relatives à la biodiversité, Revue Tiers Monde, 202, 2, 15-32.

Bonneuil, C., Fressoz, J.-B., 2013. L'événement Anthropocène. La Terre, l'histoire et nous, Paris, Seuil.

Boudia, S., Henry, E. (Eds), 2015. La mondialisation des risques. Une histoire politique et transnationale des risques sanitaires et environnementaux, Rennes, Presses universitaires de Rennes.

Bourg, D., Joly, P.-B., Kaufmann, A. (Eds), 2013. Du risque à la menace. Penser la catastrophe, Paris, Presses universitaires de France.

Caillé, A., Dufoix, S. (Eds), 2013. Le tournant global des sciences sociales, Paris, La Découverte.

Chartier, D., Deléage, J.-P., 2010. Mise à jour des écologies politiques pour une politique de l'anthropocène, Écologie $\mathcal{E}$ politique, 40, 15-20.

Cournil, C., Vlassopoulos, C. (Eds), 2015. Mobilité humaine et environnement. Du global au local, Versailles, Quæ.

Dahan, A., (Ed.), 2007. Les modèles du futur. Changement climatique et scénarios économiques: enjeux scientifiques et politiques, Paris, La Découverte.

Dardot, P., Laval, C., 2014. Commun. Essai sur la révolution au XXI siècle, Paris, La Découverte.

Décamps, H., Mathieu, N., 2005. Événements extrêmes : retours d'expérience, Natures Sciences Sociétés, 13, 4, 369-370.

Deldrève, V., Candau, J., 2015. Inégalités intra et intergénérationnelles à l'aune des préoccupations environnementales, Revue française des affaires sociales, 1-2, 79-98.

DeLoughrey, E., Didur, J., Carrigan, A. (Eds), 2015. Global ecologies and the environmental humanities. Postcolonial approaches, New York/London, Routledge.
Descola, P., 2011. L'écologie des autres. L'anthropologie et la question de la nature, Versailles, Quæ.

Deverre, C., Lamine, C., 2010. Les systèmes agroalimentaires alternatifs. Une revue de travaux anglophones en sciences sociales, Économie rurale, 317, 57-73.

Dubuisson-Quellier, S., 2014. Les engagements et les attentes des consommateurs au regard des nouveaux modes de consommation : des opportunités pour l'économie circulaire, Responsabilité et environnement, 76, 28-32.

Encinas de Munagorri, R. (Ed.), 2009. Expertise et gouvernance $d u$ changement climatique, Paris, LGDJ.

Flipo, F., 2014. Nature et politique. Contribution à une anthropologie de la modernité et de la globalisation, Paris, Éditions Amsterdam.

Forsyth, T., 2003. Critical political ecology. The politics of environmental science, New York/London, Routledge.

Foucault, M., 2001. La « gouvernementalité », in Foucault, M., Dits et écrits, 1954-1988. II, 1976-1988, Paris, Gallimard, 635657.

Foyer, J., 2010. Il était une fois la bio-révolution. Nature et savoirs dans la modernité globale, Paris, Presses universitaires de France.

Fressoz, J.-B., Graber, F., Locher, F., Quenet, G., 2014. Introduction à l'histoire environnementale, Paris, La Découverte.

Gadéa, C., 2015. Logiques professionnelles et problématiques environnementales, SociologieS, http://sociologies.revues.org/ 5113.

Gadrey, J., 2015. Adieu à la croissance. Bien vivre dans un monde solidaire, Paris, Les Petits matins.

Garrard, G., Handwerk, G., Wilke, S. (Eds), 2014. Imagining anew: challenges of representing the Anthropocene, Environmental Humanities, 5, 149-232.

Gaudillière, J.-P., Flipo, F., 2009. Inégalités écologiques, croissance « verte » et utopies technocratiques, Mouvements, 60, 77-91.

Gauthier-Clerc, M., Mesléard, F., Blondel, J. (Eds), 2014. Sciences de la conservation, Louvain-la-Neuve, De Boeck.

Gay, H., 2013. The Silwood circle. A history of ecology and the making of scientific careers in late twentieth-century Britain, London, Imperial College Press.

Gemenne, F. (Ed.), 2015. L'enjeu mondial. L'environnement, Paris, Presses de Sciences Po.

Giddens, A., 2011. The politics of climate change, Cambridge, Polity Press.

Granjou, C., 2013. Micropolitiques de la biodiversité. Experts et professionnels de la nature, Bruxelles, Peter Lang.

Grevsmühl, S.V., 2014. La Terre vue d'en haut. L'invention de l'environnement global, Paris, Seuil.

Guégan, J.-F., Renaud, F., 2005. Vers une écologie de la santé, in Barbault, R., Chevassus-au-Louis, B. (Eds), Biodiversité et changements globaux. Enjeux de société et défis pour la recherche, Paris, Association pour la diffusion de la pensée française/ministère des Affaires étrangères, 100-116.

Hache, É., 2011. Ce à quoi nous tenons. Propositions pour une écologie pragmatique, Paris, La Découverte.

Hache, É. (Ed.), 2014. De l'univers clos au monde infini, Bellevaux, Éditions Dehors.

Harribey, J.-M., 2013. La richesse, la valeur et l'inestimable. Fondements d'une critique socio-écologique de l'économie capitaliste, Paris, Les liens qui libèrent. 
Heinich, N., 2004. La sociologie de l'art, Paris, La Découverte.

Hermitte, M.-A., 2011. La nature, sujet de droit ?, Annales, 1, 173-212.

Hornborg, A., McNeill, J.R., Martinez-Alier, J. (Eds), 2007. Rethinking environmental history. World-system history and global environmental change, Lanham/Plymouth, AltaMira Press.

Humbert, M., Viveret, P., Latouche, S., Caillé, A., 2011. De la convivialité. Dialogues sur la société conviviale à venir, Paris, La Découverte.

Kalaora, B., Vlassopoulos, C., 2013. Pour une sociologie de l'environnement. Environnement, société et politique, Seyssel, Champ Vallon.

Jasanoff, S., 2005. Designs on nature. Science and democracy in Europe and the United States, Princeton, Princeton University Press.

Jasanoff, S., 2011. Cosmopolitan knowledge: climate science and global civic epistemology, in Dryzek, J.S., Norgaard, R.B., Schlosberg, D. (Eds), The Oxford handbook of climate change and society, New York, Oxford University Press, 129143.

Jasanoff, S., Martello, M.L. (Eds), 2004. Earthly politics. Local and global in environmental governance, Cambridge/London, The MIT Press.

Labatut, J., 2010. Construire la biodiversité. Processus de conception de «biens communs », Paris, Presses des mines.

Lagadeuc, Y., Chenorkian, R., 2009. Les systèmes socioécologiques: vers une approche spatiale et temporelle, Natures Sciences Sociétés, 17, 2, 194-196.

Larrère, C., Larrère, R., 2015. Penser et agir avec la nature. Une enquête philosophique, Paris, La Découverte.

Lascoumes, P., 2012. Action publique et environnement, Paris, Presses universitaires de France.

Latour, B., 2012. Enquête sur les modes d'existence. Une anthropologie des Modernes, Paris, La Découverte.

Leca, J., 1996. Ce que l'analyse des politiques publiques pourrait apprendre sur le gouvernement démocratique, Revue française de science politique, 46, 1, 122-133.

Le Treut, H., 2009. Nouveau climat sur la Terre: comprendre, prédire, agir, Paris, Flammarion.

Löwy, M., 2008. Crise écologique, capitalisme et altermondialisme. Un point de vue éco-socialiste, Actuel Marx, 44, 68-75.

Mace, G., 2013. Ecology must evolve, Nature, 503, 191-192.

Macnaghten, P., Urry, J. (Eds), 1998. Contested natures, London, Sage.

Mahrane, Y., Fenzi, M., Pessis, C., Bonneuil, C., 2012. De la nature à la biosphère. L'invention politique de l'environnement global, 1945-1972, Vingtième siècle, 113, 127-141.

Mairet, G., 2012. Nature et souveraineté. Philosophie politique en temps de crise écologique, Paris, Presses de Sciences Po.
Marcus, G.E., 1995. Ethnography in/of the world system: the emergence of multi-sited ethnography, Annual Review of Anthropology, 24, 95-117.

Maris, V., 2010. Philosophie de la biodiversité. Petite éthique pour une nature en péril, Paris, Buchet-Chastel.

Martinez-Alier, J., 2002. The environmentalism of the poor. A study of ecological conflicts and valuation, Northampton, Edward Elgar.

Micoud, A., 2005. La biodiversité est-elle encore naturelle?, Écologie E politique, 30, 17-25.

Mol, A.P.J., Sonnenfeld, D.A., Spaargaren, G. (Eds), 2009. The ecological modernisation reader. Environmental reform in theory and practice, New York/London, Routledge.

Muller, P., 2013. Les politiques publiques, Paris, Presses universitaires de France.

Petit, O., Hubert, B., Theys, J., 2014. Science globale et interdisciplinarité : quand contagion des concepts rime avec confusion, Natures Sciences Sociétés, 22, 3, 187-188.

Poirot-Delpech, L., Raineau, S. (Eds), 2012. Pour une socioanthropologie de l'environnement. Tome 1. Par-delà le local et le global, Paris, L'Harmattan.

Pries, L., 2005. Configurations of geographic and societal spaces: a sociological proposal between 'methodological nationalism' and the 'spaces of flows', Global Networks, 5, 2, 167-190.

Revel, J. (Ed.), 1996. Jeux d'échelles. La micro-analyse à l'expérience, Paris, Gallimard/Seuil.

Revet, S., Langumier, J. (Eds), 2013. Le gouvernement des catastrophes, Paris, Karthala.

Rumpala, Y., 2010. Développement durable. Ou le gouvernement du changement total, Lormont, Le Bord de l'eau.

Sinaï, A. (Ed.), 2013. Penser la décroissance. Politiques de l'Anthropocène, Paris, Presses de Sciences Po.

Sinaï, A. (Ed.), 2015. Économie de l'après-croissance. Politiques de l'Anthropocène II, Paris, Presses de Sciences Po.

Selmi, A., Hirtzel, V. (Eds), 2007. Gouverner la nature, Paris, L'Herne.

Szerszynski, B., Urry, J. (Eds), 2010. Changing climates: introduction, Theory, Culture \& Society, 27, 2-3, 1-8.

Theys, J., 2011. Les villes « post-carbone » moteurs de l'économie verte de demain?, Responsabilité et environnement, 61, 128-133.

Urry, J., 2011. Climate change and society, Cambridge, Polity Press.

Vadrot, A.B.M., 2014. The politics of knowledge and global biodiversity, New York/London, Routledge.

Yearley, S., 2005. Cultures of environmentalism. Empirical studies in environmental sociology, Basingstoke, Palgrave/ Macmillan.

Zaccai, E., Gemenne, F., Decroly, J.-M. (Eds), 2012. Controverses climatiques, sciences et politique, Paris, Presses de Sciences Po. 